\title{
Systematische Entwicklung eines Lerntools zur Erhöhung der Argumentationsfähigkeiten von Studierenden
}

\author{
Thiemo Wambsganss ${ }^{1}$, Florian Weber ${ }^{2}$, Tobias Staufenberg ${ }^{2}$, Leopold Bott ${ }^{2}$, Matthias \\ Söllner ${ }^{1,2}$ \\ ${ }^{1}$ University of St.Gallen (HSG), Institute of Information Management, St.Gallen, Switzerland \\ \{thiemo.wambsganss, matthias.soellner\}@unisg.ch \\ ${ }^{2}$ University of Kassel, Information Systems and Systems Engineering, Kassel, Germany \\ \{florianweber, leopold.bott, tobias.staufenberg\}@ student.uni-kassel.de, soellner@uni-kassel.de
}

\begin{abstract}
Die Digitalisierung führt zu neuen Anforderungen an Fähigkeiten und Kenntnissen, die Studierende in ihrem zukünftigen Berufsleben benötigen. Metakognitive Lernkompetenzen und Higher Order Thinking Skills werden dabei immer wichtiger, um Herausforderungen der Zukunft zu lösen. Eine Unterklasse dieser Fähigkeiten, die wesentlich zu Kommunikation, Kollaboration und Problemlösung beiträgt, ist die Fähigkeit, strukturiert und reflektierend $\mathrm{zu}$ argumentieren. Bildungseinrichtungen haben jedoch Schwierigkeiten, die für die Entwicklung dieser Fähigkeit notwendigen Rahmenbedingungen zu schaffen. In diesem Paper stellen wir unser Design Science Research Projekt vor, in dem wir ein Lerntool entwickeln, dass es ermöglicht, Studierende durch intelligentes Feedback beim Erlernen von Argumentation zu unterstützen. Wir präsentieren dazu acht Designprinzipien, abgeleitet aus aktueller Literatur und aus 21 Nutzerinterviews, sowie einen evaluierten Prototyen als eine Form der Instanziierung dieser Prinzipien. Ziel unseres Forschungsprojektes ist es, ein Lerntool $\mathrm{zu}$ entwickeln, das Studierenden hilft, ihre Argumentationsfähigkeit durch individuelles Feedback zu verbessern.
\end{abstract}

Keywords: Argumentation Learning, Argumentation Tool, Collaborative Learning, Technology-Based Learning Systems, Intelligent Tutoring Systems

\section{$1 \quad$ Einleitung}

Faktenwissen kann heute zunehmend über technische Hilfsmittel abgerufen werden. Dies führt dazu, dass Fähigkeiten und Kompetenzen, die über die reine Reproduktion von Wissen hinausgehen, immer wichtiger werden. Deutlich wird dies in einer Verschiebung der Berufsbilder hin zu interdisziplinären, mehrdeutigen und kreativen Aufgaben [1]. Bildungseinrichtungen sind deshalb gefordert, ihre Lehre, insbesondere in Bezug auf die Zusammensetzung und des Vermittelns von Fähigkeiten, ständig weiterzuentwickeln. Besonders die Vermittlung höherwertiger Denkfähigkeiten (Higher Order Thinking Skills), wie z. B. kritisches Denken, Kollaboration oder

$15^{\text {th }}$ International Conference on Wirtschaftsinformatik,

March 08-11, 2020, Potsdam, Germany 
Problemlösung, hat an Bedeutung gewonnen [2]. Dies wurde bereits von der Organisation für wirtschaftliche Zusammenarbeit und Entwicklung (OECD) erkannt, welche diese Fähigkeiten als wesentliches Element in ihren Learning Framework 2030 [3] aufgenommen hat.

Eine Unterklasse dieser Kompetenzen ist die Fähigkeit, strukturiert, reflektierend und gut geformt zu argumentieren [4]. Argumentation ist nicht nur ein wesentlicher Bestandteil unserer täglichen Kommunikation und unseres Denkens, sondern trägt auch wesentlich zu den Kompetenzen Kommunikation, Kollaboration und Problemlösung bei [5]. Ausgehend von ersten Studien des Aristoteles, wird die Fähigkeit strukturierte Argumente zu formulieren als Grundlage für die Überzeugung einer Zuhörerschaft gesehen und spielt eine wichtige Rolle bei strategischen Entscheidungsfindungen, bspw. bei der Analyse unterschiedlicher Standpunkte.

Um jedoch Fähigkeiten wie Argumentation zu entwickeln, ist es für den einzelnen Lernenden von großer Bedeutung, während seiner Lernentwicklung kontinuierliches Feedback zu erhalten [6, 7]. Bildungseinrichtungen, wie Universitäten, stehen vor der Herausforderung, solche Lernbedingungen zu schaffen, da es durch die steigende Zahl an Studierenden, mit gleichzeitig abnehmenden finanziellen Mitteln, immer mehr Massenvorlesungen gibt. Die von der OECD vorgelegten Zahlen spiegeln diese Entwicklung wider. Demnach stieg die Zahl der Studierenden an Hochschulen in den USA um 15 und in Deutschland um 29 Prozentpunkte, während die öffentlichen Bildungsausgaben in den USA um sieben Prozentpunkte und in Deutschland zwischen 2005 und 2014 um einen Prozentpunkt sanken [8].

Unter Berücksichtigung dieser organisatorischen und wirtschaftlichen Grenzen scheint der effektive Einsatz von Informationstechnologien (IT) ein vielversprechender Ansatz $\mathrm{zu}$ sein, um die Vermittlung von Argumentationsfähigkeiten in großen Lehrveranstaltungen individuell zu verbessern. Eine Lösung könnte die Verwendung einer adaptiven technologiebasierten Anwendung in der Lernentwicklung eines Studierenden sein, bspw. ein Intelligent Tutoring Systems (ITS). Forscher, insbesondere aus dem Bereich Educational Technology, haben dazu verschiedene Ansätze entwickelt, um die Lehre von Argumentation für Studierende oder Schüler aktiv mit Eingabemasken oder Repräsentationsrichtlinien zu unterstützen und das Erlernen von Argumentation zu verbessern [9-11]. Erste Designprinzipien für ein Lerntool, welches individuelles Feedback auf die Argumentationsfähigkeiten von Studierenden gibt, haben Wambgsanss und Rietsche 2019 beschrieben [12]. In der aktuellen Literatur gibt es jedoch nur wenige Ansätze, welche solche Designprinzipien für die Entwicklung eines adaptiven und intelligenten Lerntools präsentiert und evaluiert, die Studierende mit intelligentem und formativem Feedback beim Erlernen von Argumentation unterstützten. Daher möchten wir mit unserem Paper einen Beitrag zu Literatur und Praxis leisten, indem wir die folgende Forschungsfrage beantworten:

RQ: Was sind die zu berücksichtigenden Anforderungen beim Entwickeln eines Lerntools, welches Studierende unterstützt, besser Argumentieren zu lernen?

Um die genannte Forschungsfrage zu beantworten, verfolgen wir den Design Science Research (DSR) Ansatz von Hevner et al. [13]. Wie bereits erwähnt, fehlt es an Gestaltungswissen für die Entwicklung von Lerntools zur Vermittlung von 
Argumentationsfähigkeiten. Der DSR-Ansatz eignet sich besonders gut, um solche Forschungslücken zu schließen. Wir beabsichtigen, ein einfaches IT-Lern-Artefakt iterativ zu entwerfen und zu evaluieren [14]. Ziel ist es, mit diesen Erkenntnissen Wissenschaftler beim Erstellen gleicher oder ähnlicher Artefakte zu unterstützen [13]. Nach unserem Wissen gibt es keine Studie, die konsequent sowohl von der wissenschaftlichen Literatur als auch von potenziellen Anwendern Anforderungen an die Entwicklung eines adaptiven Lerntools für Argumentationsfähigkeiten ableitet. Unter dem Begriff adaptiven Lerntool verstehen wir eine Anwendung, welche Lernenden individuelles Feedback zu einem gegebenen Text liefert.

Im Folgenden werden wir den Leser zunächst in den notwendigen theoretischen Hintergrund einführen. Anschließend präsentieren wir unseren methodischen Ansatz für die Entwicklung des Artefakts anhand des Three-Cycle-Views von Hevner [15]. Danach legen wir die Ergebnisse der ersten sechs Schritte unseres Forschungsprojektes dar. Dies geschieht durch die Beschreibung unseres Artefakts, der Präsentation der Evaluation und das Diskutieren der Ergebnisse.

\section{Theoretische Einordnung}

\subsection{Lernen von Argumentationsfähigkeiten}

Argumentation ist eine allgegenwärtige Grundlage unserer täglichen Kommunikation und unseres Denkens. Im Allgemeinen zielt sie darauf ab, die Akzeptanz eines umstrittenen Standpunktes zu erhöhen oder zu verringern [16]. Logisch strukturierte Argumente sind eine notwendige Voraussetzung für überzeugende Gespräche, allgemeine Entscheidungen und Schlussfolgerungen. Im Kontext der Digitalisierung wird die Argumentationsfähigkeit für eine erfolgreiche Zusammenarbeit in fast jedem Beruf immer wichtiger, da sich Berufsbilder in Richtung interdisziplinärer, mehrdeutiger und kreativer Aufgaben verschieben [1]. Dies wurde auch von der OECD erkannt, welche sogenannten metakognitive Kompetenzen zu einem wesentlichen Bestandteil ihres Learning Framework 2030 [3] erklärte.

Nicht nur in der Praxis, sondern auch in der Forschung zeigen Studien, dass Argumentation für das wissenschaftliche Denken zentral ist [17-19]. Wie von Aufschnaiter et al. [20] beschrieben, nutzen Wissenschaftler Argumentation, um ihre wissenschaftlichen Aussagen zu artikulieren, zu verfeinern und zu diskutieren. Wie Kuhn [18] feststellt, ist die Argumentationsfähigkeit nicht nur für berufliche Zwecke wie Kommunikation, Zusammenarbeit und die Lösung schwieriger Probleme von großer Bedeutung, sondern auch für den größten Teil unseres täglichen Lebens.

Das Lehren von Argumentationsfähigkeiten ist jedoch begrenzt. Jonassen und Kim [21] identifizierten dafür drei Hauptursachen: Lehrenden fehlen häufig die pädagogischen Fähigkeiten, Argumentation zu fördern. Zusätzlich mangelt es an Übungsmöglichkeiten, Argumentation zu praktizieren. Außerdem führt externer Druck, viele Lehrinhalte in kurzer Zeit zu vermitteln, dazu, dass es keine Zeit für die individuelle Entwicklung der Fähigkeiten Lernender gibt.

Viele Autoren fordern deshalb, dass der Förderung von Argumentationsfähigkeiten eine zentralere Rolle in unserem formalen Bildungssystem zukommen sollte [22, 23]. 
Die meisten Schüler lernen Argumentation meist nur durch die Interaktion mit ihren Klassenkameraden oder Lehrern. Tatsächlich fehlt es in den meisten LehrLernszenarien an individueller Unterstützung der Lernenden. Um jedoch Fähigkeiten wie Argumentation zu entwickeln, ist es für den einzelnen Lernenden von großer Bedeutung, dass er während seiner Lernentwicklung kontinuierliches Feedback erhält [7]. Laut Sadler [24] ist das Ergebnis eines solchen Feedbacks eine spezifische Information über die Aufgabe oder den Lernprozess, die eine Lücke zwischen dem, was verstanden wird und dem, was verstanden werden soll, schließt. Selbst in Bereichen, in denen Argumentation Teil des Lehrplans ist, wie z. B. Recht und Logik, ist die Fähigkeit eines Lehrers, Feedback zu geben, durch Zeit- und Verfügbarkeit eingeschränkt [25]. Gerade in immer häufiger werdenden Massenvorlesungen wird die Möglichkeit, Argumentationsfähigkeiten eines Studierenden individuell zu unterstützen, unmöglich, da es für Lehrer und Professoren immer schwieriger wird, einem einzelnen Studierenden kontinuierliches und individuelles Feedback zu geben.

\subsection{Technologiebasierte Lernsysteme für Argumentationsfähigkeiten}

Die Anwendung von IT im Bildungswesen bringt mehrere Vorteile mit sich: Konsistenz, Skalierbarkeit, wahrgenommene Fairness und bessere Verfügbarkeit im Vergleich zu menschlichen Tutoren. IT-gestützte Argumentationslernsysteme könnten dazu beitragen, einen Teil der Belastung für Lehrer beim Vermitteln von Argumenten zu verringern, indem sie Lernende bei der Erstellung, Bearbeitung, Interpretation oder Überprüfung von Argumenten unterstützen [26].

Koschmann [27] unterschied zwischen vier Hauptparadigmen im Bereich der Bildungstechnologie. Jedes Paradigma enthält spezifische Annahmen über Lernen und Lehren und verwendet unterschiedliche Technologie- und Forschungsansätze. Es wird unterschieden zwischen Computer-Assisted Instruction (CAI), Intelligent Tutoring Systems (ITS), Logo-as-Latin, und Computer-Supported Collaborative Learning (CSCL) [27]. Die beiden Paradigmen CSCL und ITS sind für das Argumentationslernen von besonderer Bedeutung [25], da argumentative Diskussionen und Debatten als Schlüssel für kollaboratives Lernen identifiziert wurden. Argumentationslernen hat sich daher als Schwerpunkt in der CSCL entwickelt. ITS konzentriert sich mehr auf die Analyse, Modellierung und Unterstützung von IT-basierten Lernaktivitäten in bestimmten Bereichen. Ein relativ neues Forschungsgebiet ist die Kombination von CSCL und ITS, um Zusammenarbeit und Argumentation adaptiv und individuell zu unterstützen [28]. Forscher, insbesondere aus dem Bereich der Bildungstechnologien, haben Lerntools entwickelt, um den aktiven Schreibprozess von Lernenden mit einer Eingabemaske oder Repräsentationsrichtlinien zu unterstützen, um das Erlernen von Argumentation zu verbessern. Dies wurde in einer Vielzahl von Bereichen untersucht, darunter Recht [9], Wissenschaft [10, 29] und Gesprächsargumentation [11].

Adaptive Unterstützungsansätze für das Erlernen von Argumentation [9, 30-32] beschreiben ein relativ neues Feld des Unterstützens von Argumentationsfähigkeiten. Ziel ist es, pädagogisches Feedback auf das Handeln und die Leistungen eines Lernenden zu geben. Dabei sollen Hinweise und Empfehlungen zur Förderung und 
Steuerung zukünftiger Aktivitäten im Schreibprozess erfolgen. Dies soll durch eine automatisierte Bewertung geschehen, die feststellt, ob ein Argument oder eine Argumentationskette syntaktisch und semantisch korrekt ist. Wie Scheuer und Lippi und Torroni [25, 33] feststellten, fehlt es hier aber an empirischer Forschung, die das Gestalten und den Einsatz solcher Systeme untersucht und evaluiert. Genau diese Forschungslücke möchten wir mit unserer Forschung schließen.

\section{Forschungsmethode}

Um unsere Forschungsfrage zu beantworten, folgen wir dem Three-Cycle-View des DSR-Ansatzes nach Hevner et al. [13]. Abbildung 1 zeigt die drei Zyklen nach Hevner [15] und unsere sechs Schritte der Durchführung.

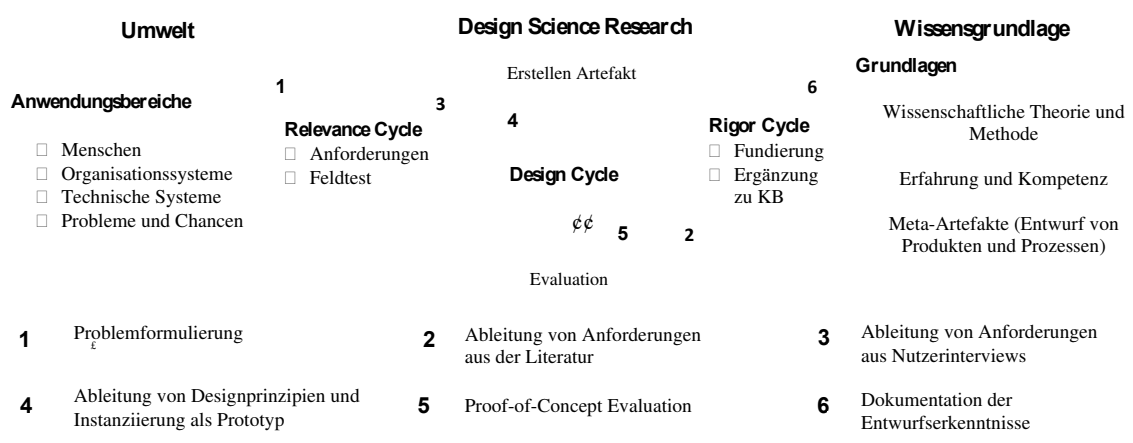

Abbildung 1. Eigene Darstellung Design Science Three-Cycle-Views nach Hevner [15]

Der erste Schritt innerhalb des DSR besteht darin, eine Problemstellung zu formulieren. Daher haben wir das Problem in der Einleitung ausführlich hergeleitet und in dem theoretischen Hintergrund erklärt. Der zweite Schritt beinhaltet die Ableitung von Anforderungen aus der wissenschaftlichen Literatur und stellt die Umsetzung des Rigor Cycles dar. Dafür haben wir wissenschaftliche Literatur aus den Bereichen Educational Technology und Lerntheorien untersucht, um Anforderungen für das Entwerfen eines Lerntools für Argumentationsfähigkeiten abzuleiten. Diese sind in Abbildung 2 als Meta Requirements (MR) dargestellt. Im dritten Schritt haben wir Experteninterviews durchgeführt. Diese hatten das Ziel, Sachinformationen mittels vom Interviewer geführten, semi-strukturierten Interviews herauszufinden. Sie basiert auf den konzeptionellen Grundlagen von qualitativen Experteninterviews nach Gläser und Laudel [34]. Insgesamt haben wir 21 Interviews mit Masterstudierenden unterschiedlicher Fachbereiche und Studiengänge durchgeführt. Die Teilnehmer waren alle potentielle Endnutzer für das Lernen von Argumentation im universitären Kontext. Aus den Interviews haben wir anschließend User Stories (US) und User Requirements (UR) abgeleitet. Im vierten Schritt haben wir auf Grundlage der vorherigen Anforderungen vorläufige Designprinzipien (DP) nach den Formulierungsvorschlägen von Chandra et al. [35] verfasst. Die US, UR und MR wurden dazu zusammengeführt, gebündelt und bildeten die Grundlage für das weitere 
Vorgehen [35]. Anschließend haben wir eine erste Instanziierung der Designprinzipien als Designfeature (DF) in einem prototypischen Mock-up umgesetzt. Im fünften Schritt haben wir eine Proof-of-Concept-Evaluierung auf der Grundlage der von Venable et al. [36] vorgeschlagenen Bewertungskriterien durchgeführt. Ziel dieser Evaluation war es, zu sehen, wie potentielle Endnutzer den Wert unserer prototypisch instanziierten Designprinzipien wahrnehmen und bewerten. Durch die Evaluation konnten wir schließlich Anpassungsvorschläge für unsere Designprinzipien ableiten und ein weiteres Designprinzip formulieren. Der sechste Schritt beinhaltet die Dokumentation des Designwissens, welches wir durch diesen Beitrag erfüllen.

\section{Design und Evaluierung des Artefakts}

In diesem Abschnitt beschreiben wir, wie wir die Anforderungen für unser Lerntool für Lernen von Argumentation gesammelt und die vorläufigen Designprinzipien hergeleitet haben. Abbildung 2 erläutert die Schritte der Abstraktion von den US und den Literatur Issues (LI) über die $U R$ und $M R$ zu den vorläufigen Designprinzipien. Die Designprinzipien werden anschließend durch Designfeature in einem ersten Prototyp veranschaulicht (Abbildungen 3) und evaluiert.

\subsection{Schritt 2: Ableitung von Anforderungen aus der Literatur}

Die Ableitung der MR aus der Theorie erfolgt im Rigor Cycle und gibt den aktuellen Stand der Forschung, vor allem im Bereich Educational Technology und Lerntheorien, wieder. Dazu wurde eine systematische Literaturrecherche nach den methodischen Ansätzen von Cooper [37] und vom Brocke et al. [38] durchgeführt. Darauf aufbauend haben wir (1) den Umfang der Recherche definiert, (2) das Thema konzeptualisiert, (3) die Literatur durchsucht und (4) die Ergebnisse bezüglich unserer Anforderungen analysiert. In Schritt 1 (Definition des Rechercheumfangs) haben wir definiert, dass wir uns zunächst auf Forschungsergebnisse konzentrieren, die die erfolgreiche Implementierung von Lerntools für Argumentationsfähigkeiten zeigen. Darüber hinaus ist es unser Ziel, Anforderungen auf konzeptioneller Ebene zu identifizieren, wobei der Schwerpunkt auf einer repräsentativen Abdeckung liegt [37]. In Schritt 2 (Konzeptualisierung des Themas) haben wir zwei große Bereiche für die Ableitung von Anforderungen identifiziert: Educational Technology und Lerntheorien. Da das Erstellen eines Lerntools für Argumentationsfähigkeiten ein komplexes Unterfangen ist, das von Psychologen, Pädagogen und Informatikern mit unterschiedlichen Methoden untersucht wird [25], haben wir uns zunächst auf diese Literaturströme konzentriert. Für Schritt 3 (Literatursuche) haben wir eine Stichwortsuche bei Google Scholar durchgeführt, um relevante Publikationen zu identifizieren. Wir haben Google Scholar verwendet, weil diese Web-Suchmaschine eine erweiterte Volltextsuche und mehrere Filteroptionen für wissenschaftliche Literatur ermöglicht. Für die Suche haben wir folgende Suchbegriffe verwendet: „Argumentation Learning”, „Argumentation Model”, „Collaborative Learning”, „ICT 
Learning”, „Learning Theory“, „Learning Tool” sowie “Learning Systems”. Dadurch erhielten wir initial über 1000 Beiträge.

Anschließend haben wir Ein- und Ausschlusskriterien definiert und Titel und Abstract der Suchergebnisse überprüft, um die Relevanz dieser Beiträge für unser Projekt zu bewerten. Wir haben nur Literatur aufgenommen, die sich mit einer Art Lerntool im Bereich Argumentationslernen beschäftigt oder dazu einen Beitrag liefert, wie bspw. durch eine etablierte Lerntheorie. Zahlreiche Beiträge, die sie sich mit Argumentation in anderen Forschungsbereichen auseinandergesetzt haben, wurden ausgeschlossen. Auf dieser Grundlage haben wir 67 Paper ausgewählt, die wir intensiver analysierten. In Bezug auf Schritt 4 (Literaturanalyse) haben wir dazu ähnliche Themen dieser Beiträge als Literatur Issues (LIs) zusammengefasst und daraus sieben Cluster gebildet.

Das erste Cluster befasst sich mit den Theorien zum Testing Effect (LI1). Hier werden positive Auswirkungen von wiederholtem praktischem Üben auf das Lernen nachgewiesen. Durch das Wiederholen von bestimmten Aufgaben und Aktivitäten verbessert sich die Abrufbarkeit von angewendeten Fähigkeiten und Wissen. Die Verbesserungen lassen sich auf testgestütztes und praktisches Anwenden zurückführen [39, 40]. Diese Erkenntnisse werden genutzt, indem das Tool wiederholte und praktische Anwendungsmöglichkeiten zur Verfügung stellen sollte. Durch diese kann der Nutzer seine Fähigkeiten aktiv trainieren und langfristig verinnerlichen [39]. Die Theorie des Multimedia Learnings von [41] (LI5) beschreibt die positiven Auswirkungen von auditivem und visuellem Wahrnehmen und Verarbeiten von Informationen auf den Lernerfolg [41]. Durch die visuelle und auditive Darstellung des Feedbacks im Tool (MR5) soll ein nachhaltiger Lerneffekt für die Nutzer erreicht werden. Weiterhin soll dadurch die kognitive Last des Nutzers reduziert werden, da die Kapazitäten der zur Verarbeitung von Informationen durch die einzelnen Aufnahmekanäle des Menschen für Bild- und Sprachmaterial begrenzt sind [42]. Durch die Theorie des kollaborativen Lernens (LI7) werden positive Effekte des sozialen Lernens genutzt. Wissen von Anderen kann als Wissensressource verwendet werden und fördert durch den Austausch von verteiltem Wissen den Wissenszuwachs in der Gruppe [43]. Zusätzlich fördert kollaboratives Argumentieren kritisches und unabhängiges Denken und regt zu Diskussionen zwischen mehreren Teilnehmern an [44]. Daraus folgt auch, dass kollaborative Gruppen mehr Argumente, Gegenargumente und Widerlegungen für die Argumentation finden [45]. Aus diesen Erkenntnissen resultiert das $M R$ 7. Nach der Theorie ist Feedback (LI3) für die Steigerung von Higher Order Thinking Skills unerlässlich [7]. Daher sollte jeder Nutzer des Lerntools individuelles Feedback auf seine Argumentation erhalten (MR3).

Weiterhin hat das Feedback die Funktion, dem Lernenden weitere Ziele vorzugeben, die er erreichen soll, sowie seinen Fortschritt zu überwachen [7]. Somit soll das Feedback durch das Aufzeigen des Lernstandes und das Definieren von weiteren Aktivitäten die Erreichung der Ziele fördern und neue Ziele definieren. Die Theorie des Learner Control Principles (LI2) [46] besagt, dass durch den Einsatz von hypermedialen Lernumgebungen die Interaktivität der Lernumgebungen steigt und somit der Nutzer eine Kontrolle über seinen Lernfortschritt erhält. Diese Kontrolle 
steigert die Motivation des Lernenden und führt zu einer aktiven und verbesserten Informationsverarbeitung [45]. Der Lernende hat die Möglichkeit, seinen Lernprozess persönlich zu steuern und die Inhalte selber zu wählen (MR2).

\subsection{Schritt 3: Ableitungen von Anforderungen aus Nutzerinterviews}

Um weitere Anforderungen an ein Lerntool für Argumentationsfähigkeiten zu erheben, wurden 21 qualitative Interviews mit Studierenden nach der Methode von Gläser und Laudel [34] geführt. Der Interviewleitfaden besteht aus 29 Fragen und jedes Interview dauerte etwa 25 bis 50 Minuten. Die Teilnehmer wurden zu folgenden Themen befragt: Erfahrung mit technologiebasierten Lernsystemen, Wahrnehmung mit bestehenden, genutzten Lernsystemen, Wichtigkeit von Fähigkeiten in der universitären Ausbildung, Anforderungen an ein System, das unterstützt, Fähigkeiten zu erlernen (z. B. Funktionalitäten, Design), Anforderungen an ein System, das unterstützt, Argumentation zu erlernen (z. B. Funktionalitäten, Design). Die Befragten waren eine zufällige Teilmenge der Studentenpopulation an unserer Universität. Um Eindrücke zu erhalten, die aus langjähriger Lernerfahrung resultieren, wurden ausschließlich Masterstudierende für die Interviews rekrutiert. Die befragten Studierenden waren zwischen 22 und 28 Jahre alt und hauptsächlich Studierende der Wirtschaftswissenschaften, des Wirtschaftsingenieurwesens, der Psychologie und der Informatik, 7 waren männlich, 14 weiblich. Nach einer genaueren Transkription wurden die Interviews über eine qualitative Inhaltsanalyse ausgewertet [34]. Die Interviews wurden kodiert und abstrakte Kategorien wurden gebildet. Die Kodierung wurde anhand eines open codeings durchgeführt, um ein einheitliches Kodierungssystem während der Auswertung zu bilden. Aus den ausgewerteten Interviews konnten 180 User Stories abgeleitet werden [47]. Hieraus wurden die elf wichtigsten User Stories konsolidiert und zusammengefasst. Auf der Basis der konsolidierten User Stories wurden die Nutzeranforderungen nach Chandra et al. [35] abgeleitet (vgl. Abbildung 2).

\subsection{Schritt 4: Ableitung von Design Prinzipien und Instanziierung als Prototyp}

Basierend auf unseren identifizierten Meta und User Requirements haben wir eine erste Gruppe von vorläufigen Designprinzipien formuliert. Den Studierenden war es demnach wichtig, durch repetitives und praktisches Üben zu lernen und so ihre Argumentationsfähigkeiten zu verbessern (URl). Zusammen mit der Theorie des Testing Effects (LII) und MRI ergibt sich dadurch das erste Designprinzip. Dieses beschreibt, dass das Lerntool mit Übungen ausgestattet sein sollte, um Fähigkeiten praktisch zu testen und anzuwenden (DPI). In dem anschließend entwickelten Prototyp wird DP1 in drei vorgeschlagenen Übungen veranschaulicht und als Designfeature 1-4 umgesetzt (vgl. Tabelle 1 und Abbildung 3). 


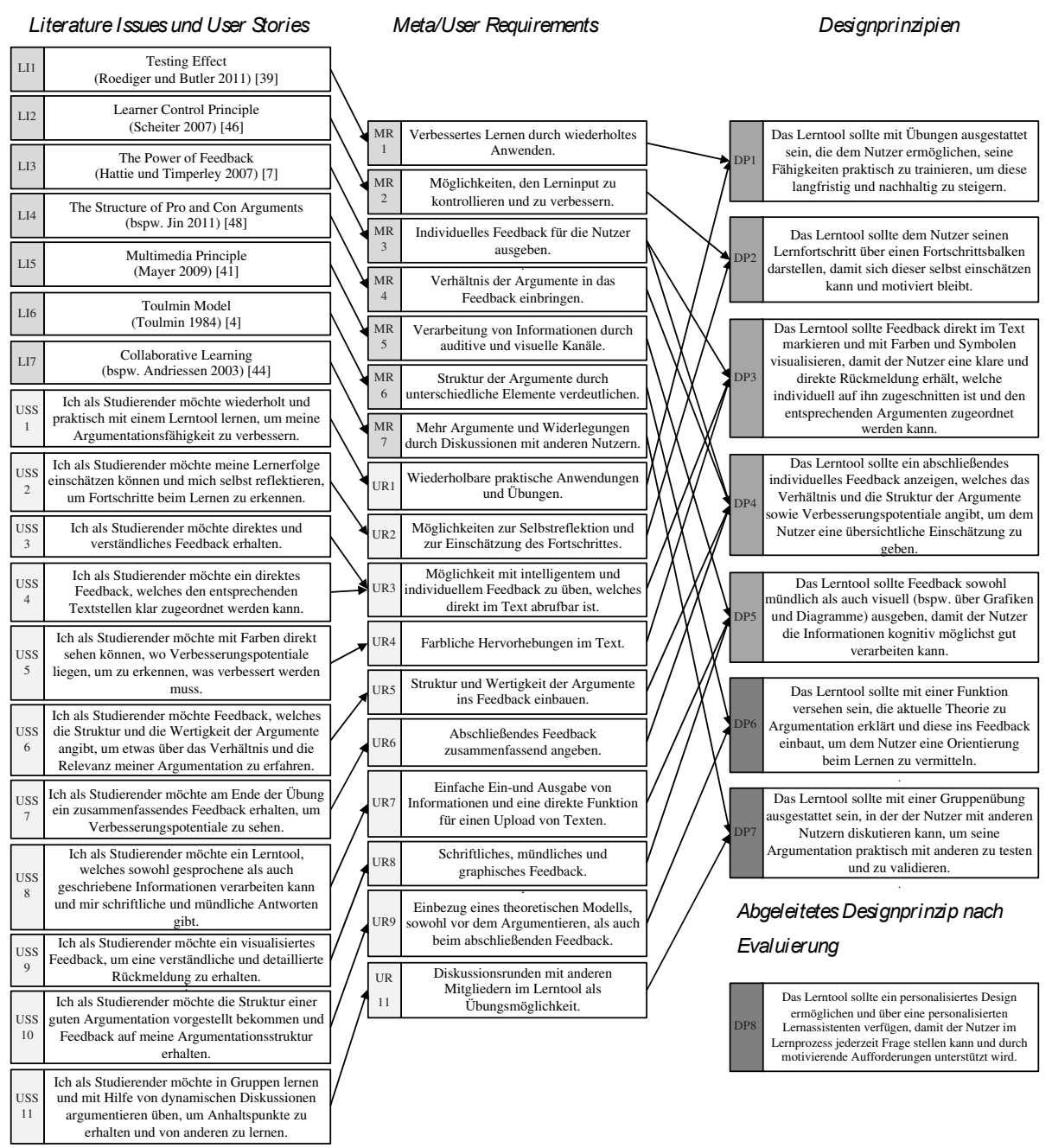

Abbildung 2. Übersicht über die vorläufigen Designprinzipien nach Chandra et al. [35]

Vielen Studierenden war es auch wichtig, sich während dem Lernen selbst reflektieren zu können und die Fortschritte beim Lernen zu überwachen (UR2). Daraus ergibt sich, dass das Lerntool eine Fortschrittsleiste enthalten sollte, welche dem Nutzer seinen Lernfortschritt anzeigt und ihn dadurch motiviert. Weiterhin gibt es dem Nutzer die Möglichkeit, sich selbst einschätzen zu können (DP2), das durch die Designfeature 5 und 6 adressiert wurde. Aus den Interviews ergibt sich zudem, dass die Studierenden sich direktes, individuelles und intelligentes Feedback wünschen (UR3). Daraus lässt sich das DP3 in Kombination mit der The Power of Feedback Theorie (LI3) ableiten. Demnach sollte das Lerntool dem Nutzer intelligentes und individuelles Feedback geben, welches den Argumenten durch Hervorhebungen direkt zugeordnet werden kann (DP3). Für die befragten 
Studierenden war ein abschließendes Feedback, welches Aussagen über die Struktur und Wertigkeit der Argumente trifft, bedeutend (UR5, UR6). Die Wertigkeit der Argumente soll über die Pro- und Kontra-Struktur der Argumente nach [48] bestimmt werden (LI4, MR4), die das Verhältnis der vorgebrachten Argumente enthält. Das daraus entstehende DP4 wird in dem Prototyp durch eine abschließende Feedbackfunktion verdeutlich, die nochmals die Wichtigkeit von individuellem und intelligentem Feedback [7] aufzeigt. Aus den Nutzerinterviews ergab sich, dass die Studierenden Feedback schriftlich, mündlich und graphisch erhalten wollen (UR8). Das Lerntool sollte daher Feedback durch Grafiken und Diagramme visuell darstellen sowie Feedback in Textform liefern. Zusätzlich sollte das schriftliche Feedback auch über einen Sprachassistenten abgerufen werden können (DP5). In Verbindung mit der Theorie des Multimedia Learnings von [41] (LI5) ergibt sich, dass die Nutzer des Lerntools das Feedback besser kognitiv verarbeiten können. Weiterhin gibt das Tool den Usern vor den Argumentationsübungen die Argumentationsstruktur nach Toulmin [4] vor (DP6). Auch kann die Argumentationsstruktur durch eine Eingabemaske in Übung 1 aktiv trainiert werden (DP1). Einigen Studierenden ist auch eine soziale Komponente beim Lernen wichtig. Sie möchten dynamische Diskussionen führen und in der Gruppe Argumente austauschen (UR 11). Daher sollte das Lerntool ermöglichen, dass die Nutzer kollaborativ lernen und mit anderem Nutzern dynamische Diskussionen führen (DP7). Dies hilft den Teilnehmern nach der kollaborativen Lerntheorie (LI7), Wissen aus der Gruppe zu ziehen und mehr stichhaltige Argumente zu sammeln [43, 45]. Jonassen und Kim [21] sehen kollaborative Argumentation auch als eine Möglichkeit an, die Fähigkeit zu stärken, Konterargumente zu entwickeln. Das DP 7 wurde durch die DF8 bis DF10 adressiert.

Tabelle 1. Instanziierung von Designprinzipien mit Designfeaturen

\begin{tabular}{|c|c|c|c|c|c|c|c|c|}
\hline \multirow{2}{*}{\multicolumn{2}{|c|}{$\begin{array}{l}\text { Designfeature der ersten Version unseres } \\
\text { Prototypen }\end{array}$}} & \multicolumn{7}{|c|}{ Adressierte Designprinzipien } \\
\hline & & \multirow{2}{*}{\begin{tabular}{|c|} 
DP1 \\
$\mathrm{X}$ \\
\end{tabular}} & \multirow[t]{2}{*}{ DP2 } & \multirow[t]{2}{*}{ DP3 } & \multirow[t]{2}{*}{ DP4 } & \multirow[t]{2}{*}{ DP5 } & \multirow[t]{2}{*}{ DP6 } & \multirow[t]{2}{*}{ DP7 } \\
\hline DF1 & $\begin{array}{l}\text { Upload Funktion von Texten, die direkt in die Übungen } \\
\text { integriert werden können. }\end{array}$ & & & & & & & \\
\hline DF2 & $\begin{array}{l}\text { Spracheingabefunktion, über die Texte eingesprochen } \\
\text { werden können. }\end{array}$ & $\mathrm{X}$ & & & & & & \\
\hline DF3 & $\begin{array}{l}\text { Eingabemaske, um die Struktur der Argumentation nach } \\
\text { Toulmin üben zu können. }\end{array}$ & $\mathrm{X}$ & & & & & $\mathrm{X}$ & \\
\hline DF4 & Nutzerfreundliche und menschenähnliche Sprache. & $\mathrm{X}$ & & $\mathrm{X}$ & $\mathrm{X}$ & $\mathrm{X}$ & & \\
\hline DF5 & Analysebericht als Feedback (Diagramme, Grafiken usw.). & & $\mathrm{X}$ & & $\mathrm{X}$ & & & \\
\hline DF6 & $\begin{array}{l}\text { Übersichtliche Funktionen mit einem funktionalen Design } \\
\text { (z. B. Icons, Bilder usw.). }\end{array}$ & & $\mathrm{X}$ & & & $\mathrm{X}$ & & \\
\hline DF7 & $\begin{array}{l}\text { Sprachausgabefunktion, die dem User das Feedback auch } \\
\text { mündlich mitteilt. }\end{array}$ & & & $\mathrm{X}$ & $\mathrm{X}$ & $\mathrm{X}$ & & \\
\hline DF8 & Chat für die Kommunikation unter den Usern. & & & & & & & $\mathrm{X}$ \\
\hline DF9 & $\begin{array}{l}\text { Text Mining Module zur adaptiven Kontrolle des } \\
\text { Lernkontextes und des Lernstandes. }\end{array}$ & & & & & & & $\mathrm{X}$ \\
\hline DF10 & $\begin{array}{l}\text { Möglichkeit andere User zu einer Argumentation } \\
\text { einzuladen. }\end{array}$ & & & & & & & $\mathrm{X}$ \\
\hline
\end{tabular}




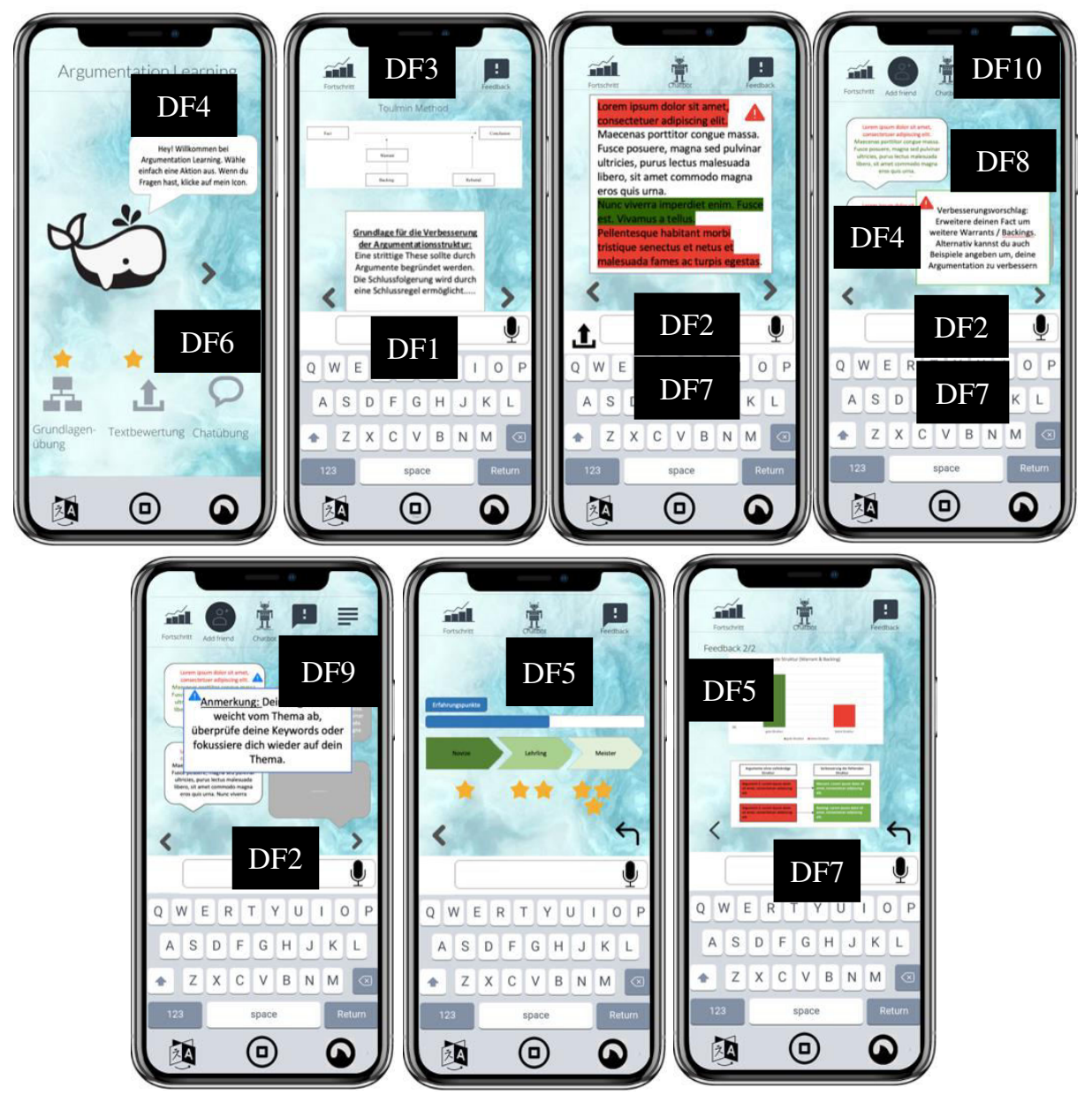

Abbildung 3. Prototypische Applikation als Instanziierung unserer Designprinzipien

In dem Prototyp des Lerntools wird dieses DP durch die Designfeature 8 bis 10 adressiert. Diese stellen einen Chat dar, in dem die Studierenden sowohl mit anderen Nutzern als auch mit einem Conversational Agent (Chatbot) diskutieren können. Der Conversational Agent soll die Argumentation des Nutzers analysieren, Schwächen identifizieren (bspw. Aussagen ohne Prämissen) und hier den Nutzer durch intelligentes Nachfragen herausfordern. Anhand eines intelligenten Systems aufbauend auf Text Mining Feature von Natural Language Processing und Machine Learning [49], bspw. Argumentation Mining [33], könnte solch ein Chatbot für das Lerntool entwickelt werden. In unserem instanziierten Prototyp ist dies jedoch nur als Mock-up umgesetzt. Der Chat bietet den Nutzern die Möglichkeit, asynchron zu diskutieren und Argumentieren zu üben. 


\subsection{Schritt 5: Proof-of-Concept Evaluation einer vorläufigen Version}

In diesem Abschnitt beschreiben wir die Proof-of-Concept Evaluierung einer ersten vorläufigen Version unseres Lerntools für Argumentation. Die Evaluation dient dazu, zu überprüfen, ob die Designprinzipien für Lernende von Nutzen sind, um dadurch eventuelle Änderungswünsche aufzunehmen. Um unsere Designprinzipien zu evaluieren, haben wir eine prototypische Applikation implementiert, welche eine Instanziierung der Designprinzipien als Designfeature darstellt. Abbildung 3 zeigt den Prototyp und wie die verschiedenen Designfeature umgesetzt wurden. Für die Erstellung des Prototypen haben wir das Onlinetool Marvel ${ }^{1}$ genutzt. Die Evaluation wurde ex ante und anhand eines „artificial“ Ansatzes durchgeführt [36]. Die Designprinzipien wurden dabei speziell anhand der Kriterien Nützlichkeit und Nutzerfreundlichkeit untersucht und anhand verschiedener Fragen evaluiert. Um dies messen zu können, wurde ein Fragebogen mit 38 Fragen erstellt und dabei die Designprinzipien anhand der oben beschrieben Punkte bewertet. Es wurden Fragen zur Wichtigkeit der Argumentationsstruktur, zur Nutzerfreundlichkeit und Nützlichkeit der einzelnen Übungen und des gesamten Lerntools gestellt. Insgesamt wurde der Fragebogen mit 32 Personen durchgeführt. Die Probanden konnten dazu den Prototyp testen. Nach dem Testen bearbeiteten die Nutzer dann den Fragebogen. Die Personengruppe setzte sich wie folgt zusammen: 22 Personen studieren im Master-Studiengang, 8 Personen befinden sich im Bachelor-Studiengang, 2 Personen gehen einer anderen Tätigkeit nach. Alle Personen waren im Alter von 22 und 34 Jahren, 19 waren weiblich, 13 männlich.

Um ein gutes und weitreichendes Feedback zu erhalten, konnten die Befragten die Nützlichkeit anhand von sieben Bewertungsstufen einordnen. Diese reichten von „völlig nützlich (1)“ bis ,völlig unnützlich (7)“ (siehe Auszug des Evaluationsbogens in Tabelle 2).

Tabelle 2. Auszug aus dem Evaluationsbogen der Proof-of-Concept Evaluierung

\begin{tabular}{|l|c|c|c|c|c|c|c|}
\hline $\mathbf{n = 3 2}$ & $\mathbf{1}$ & $\mathbf{2}$ & $\mathbf{3}$ & $\mathbf{4}$ & $\mathbf{5}$ & $\mathbf{6}$ & $\mathbf{7}$ \\
\hline Wie wichtig ist Dir eine Erklärung der & $4 \mathrm{x}$ & $16 \mathrm{x}$ & $10 \mathrm{x}$ & $2 \mathrm{x}$ & - & - & $1 \mathrm{x}$ \\
Argumentationsstruktur? & $12,2 \%$ & $48,5 \%$ & $30,3 \%$ & $6,1 \%$ & & $3,03 \%$ \\
\hline Wie wichtig ist Dir die direkte Bewertung & $12 \mathrm{x}$ & $15 \mathrm{x}$ & $3 \mathrm{x}$ & $2 \mathrm{x}$ & - & - & - \\
von Argumenten im Text? & $37,5 \%$ & $46,9 \%$ & $9,3 \%$ & $6,2 \%$ & & \\
\hline
\end{tabular}

Die Evaluation der allgemeinen Usability sowie Nützlichkeit ergab folgendes Ergebnis: Die Befragten bewerteten die allgemeine Usability der umgesetzten Designprinzipien als gut (arithm. Mittel von 2,45). Ebenso wurde das Tool als nützlich bewertet (arithm. Mittel 2,16). Die Designprinzipien wurden ebenfalls sehr positiv eingeordnet: Ein direktes Feedback im Text bewerten 96,9\% als nützlich. Eine Fortschrittseinordnung finden $93,5 \%$ wichtig.

Als Ableitung für das weitere Vorgehen lässt sich folgendes festhalten: Einige Befragte wünschen sich einen Fortschrittsstatus, der auch die zuletzt erfolgte Bewertung aufzeigt. Für die Designprinzipien ergibt sich hieraus, dass die

\footnotetext{
${ }^{1}$ https://marvelapp.com/
} 
Fortschrittsanzeige detaillierter und individueller gestaltet werden sollte. Oft gefordert wurde außerdem die Möglichkeit das Design des Lerntools zu personalisieren. Zusätzlich erwähnten einige Nutzer, dass sie gerne einen personalisierten Lernassistenten (bspw. in Form eines Conversational Agents) hätten, der ihnen jederzeit Fragen beantwortet und sie beim Lernen bspw. durch motivierende Aufforderungen unterstützt. Diese Anforderungen werden in einem achten Designprinzip in Abbildung 2 dargestellt.

\section{$5 \quad$ Diskussion und Ausblick}

In diesem Beitrag berichten wir über unser Forschungsprojekt, mit dem wir ein Lerntool entwickeln, dass Studierenden helfen soll, Argumentationsfähigkeiten zu verbessern. Wie dargestellt, wurden elf User Stories identifiziert sowie sieben vorläufige MRs und elf URs formuliert. Basierend auf diesen Ergebnissen haben wir acht vorläufige Designprinzipien für ein Lerntool zum Trainieren von Argumentationsfähigkeiten als spezielle Klasse der metakognitive Lernkompetenzen, abgeleitet. Die Designprinzipien wurden auf der Grundlage von aktueller Literatur sowie Nutzerbedürfnissen formuliert. Wir glauben, dass ein Lerntool zum Trainieren von Argumentationsfähigkeiten (und möglicherweise auch metakognitive Lernkompetenzen), welches unsere Designprinzipien instanziiert, die Motivation der Lernenden erhöhen sollte, und so das Lernergebnis verbessert. Beispielsweise sollte ein Argumentationslerntool, das sofort individuelles Feedback liefert und den Lernenden die Flexibilität gibt, ihren Lerninput $\mathrm{zu}$ kontrollieren und ihren Lernfortschritt zu überwachen, die Motivation des Nutzers erhöhen, und somit den Lernprozess signifikant beim Aufbau von neuem Wissen unterstützen.

Damit leistet unsere Arbeit mehrere Beiträge zur aktuellen Forschung. Nach bestem Wissen ist diese Studie die erste, die Designwissen darüber präsentiert, wie man ein Lerntool zum Trainieren von Argumentationsfähigkeiten entwirft. Zudem bieten wir damit eine Grundlage für Forscher, die ebenfalls Lerntools zum Trainieren von metakognitiven Kompetenzen entwickeln, ihre Lösung mit unserer zu vergleichen. Unsere Arbeit hat auch Auswirkungen auf die Praxis. Dozenten und Bildungseinrichtungen können unsere Gestaltungsprinzipien nun nutzen, um eigene Lerntools für metakognitive Lernkompetenzen zu erstellen.

Trotzdem gibt es eine Reihe von Einschränkungen in unserem Beitrag zu beachten. Zunächst haben wir Anforderungen aus einer bestimmten theoretischen Perspektive und einer bestimmten Nutzergruppe gesammelt. Es ist möglich, dass andere Bereiche der Literatur und andere Nutzergruppen zu unterschiedlichen Ergebnissen geführt hätten. Darüber hinaus konnten wir unser Lerntool noch nicht voll entwickeln, um es in einer groß angelegten Vorlesung während eines ganzen Semesters zu evaluieren. Dies hätte uns weitere Erkenntnisse über den langfristigen Einsatz eines solchen Lerntools und die Auswirkung auf den Lernerfolg von Studierenden geben können.

Für die zukünftige Forschungsarbeit empfehlen wir deshalb, größere Testgruppen zu nutzen, um noch aussagekräftigere Ergebnisse zu erhalten. Weiterhin sollte die Evaluation innerhalb einer groß angelegten Vorlesung oder eines Seminars mit einer 
vollfunktionierenden Version des Lerntools stattfinden, um Studierende direkt innerhalb des vorgesehenen Einsatzbereiches zu befragen und einen Effekt auf den tatsächlichen Lernerfolg zu messen.

\section{References}

1. vom Brocke, J., Maaß, W., Buxmann, P., Maedche, A., Leimeister, J.M., Pecht, G.: Future Work and Enterprise Systems. Bus. Inf. Syst. Eng. 60, 357-366 (2018).

2. Fadel, C., Bialik, M., Trilling, B.: Four-dimensional education : the competencies learners need to succeed. (2015).

3. OECD: The Future of Education and Skills - Education 2030, (2018).

4. Toulmin, S.E.: Introduction to Reasoning. (1984).

5. Kuhn, D.: Thinking as Argument. Harv. Educ. Rev. 62, 155-179 (1992).

6. Black, P., Wiliam, D.: Developing the theory of formative assessment. Educ. Assessment, Eval. Account. 21, 5-31 (2009).

7. Hattie, J., Timperley, H.: The Power of Feedback. Rev. Educ. Res. 77, 81-112 (2007).

8. OECD: Education at a Glance 2016: OECD Indicators. , Paris (2016).

9. Pinkwart, N., Ashley, K., Lynch, C., Aleven, V.: Evaluating an Intelligent Tutoring System for Making Legal Arguments with Hypotheticals. IOS Press (2009).

10. Osborne, J.F., Henderson, J.B., MacPherson, A., Szu, E., Wild, A., Yao, S.Y.: The development and validation of a learning progression for argumentation in science. J. Res. Sci. Teach. 53, 821-846 (2016).

11. De Groot, R., Drachman, R., Hever, R., Schwartz, B., Hoppe, U., Harrer, A., De Laat, M., Wegerif, R., Mclaren, B.M., Baurens, B.: Computer Supported Moderation of EDiscussions: the ARGUNAUT Approach. (2007).

12. Wambsganss, T., Rietsche, R.: Towards Designing an Adaptive Argumentation Learning Tool. International Conference on Information Systems (ICIS) (2019).

13. Hevner, A.R., March, S.T., Park, J., Ram, S.: Design Science in Information Systems Research. Des. Sci. IS Res. MIS Q. 28, 75 (2004).

14. Jones, D., Gregor, S.: The Anotomy of a Design Theory. J. Ass. Inf. Syst. 8, 312-335 (2007).

15. Hevner, A.R.: A three cycle view of design science research. Scand. J. Inf. Syst. 1-6 (2007).

16. Eemeren, F.H. van, Grootendorst, R., Johnson, R.H., Plantin, C., Willard, C.A., Grootendorst, R., Johnson, R.H., Plantin, C., Willard, C.A.: Fundamentals of Argumentation Theory. Routledge (1996).

17. Duschl, R.A., Osborne, J.: Supporting and Promoting Argumentation Discourse in Science Education. Stud. Sci. Educ. 38, 39-72 (2002).

18. Kuhn, D.: Science as argument: Implications for teaching and learning scientific thinking. Sci. Educ. 77, 319-337 (1993).

19. Newton, R.P., Roef, L., Witters, E., Van Onckelen, H.: Tansley Review No. 106. New Phytol. 143, 427-455 (1999).

20. Von Aufschnaiter, C., Erduran, S., Osborne, J., Simon, S.: Arguing to learn and learning to argue: Case studies of how students' argumentation relates to their scientific knowledge. J. Res. Sci. Teach. 45, 101-131 (2008).

21. Jonassen, D.H., Kim, B.: Arguing to learn and learning to argue: Design justifications and guidelines. Educ. Technol. Res. Dev. 58, 439-457 (2010). 
22. Driver, R., Newton, P., Osborne, J.: Establishing the norms of scientific argumentation in classrooms. Sci. Educ. 84, 287-312 (2000).

23. Kuhn, D.: Education for thinking. Harvard University Press (2005).

24. Sadler, D.R.: Formative assessment and the design of instructional systems. Instr. Sci.

25. Scheuer, O.: Towards adaptive argumentation learning systems. (2015).

26. Scheuer, O., Loll, F., Pinkwart, N., McLaren, B.M.: Computer-supported argumentation: A review of the state of the art. Int. J. Co. Collab. Learn., 43-102 (2010).

27. Koschmann, T.: Paradigm Shifts and Instructional Technology. Lawr. Erlbaum Ass. (1996).

28. Fischer, F., Kollar, I., Stegmann, K., Wecker, C.: Toward a Script Theory of Guidance in Computer-Supported Collaborative Learning. Educ. Psychol. 48, 56-66 (2013).

29. Suthers, D.D., Hundhausen, C.D.: European Perspectives on Computer-Supported Collaborative Learning. (2001).

30. Stab, C., Gurevych, I.: Identifying Argumentative Discourse Structures in Persuasive Essays. In EMNLP pp. 46-56 (2014).

31. Stab, C., Gurevych, I.: Parsing Argumentation Structures in Persuasive Essays. Comput. Linguist. 43, 619-659 (2017).

32. Huang, C.J., Chang, S.C., Chen, H.M., Tseng, J.H., Chien, S.Y.: A group intelligencebased asynchronous argumentation learning-assistance platform. Interact. Learn. Environ. 24, 1408-1427 (2016).

33. Lippi, M., Torroni, P.: Argumentation Mining: State of the Art and Emerging Trends. IJCAI Int. Jt. Conf. Artif. Intell., 4207-4211 (2015).

34. Gläser, J., Laudel, G.: Experteninterviews und qualitative Inhaltsanalyse : als Instrumente rekonstruierender Untersuchungen. VS Verlag für Sozialwiss (2010).

35. Chandra, L., Seidel, S., Gregor, S.: Prescriptive knowledge in IS research: Conceptualizing design principles in terms of materiality, action, and boundary conditions. Proc. Annu. Hawaii Int. Conf. Syst. Sci. 2015-March, 4039-4048 (2015).

36. Venable, J., Pries-Heje, J., Baskerville, R.: FEDS: A Framework for Evaluation in Design Science Research. Eur. J. Inf. Syst. 25, 77-89 (2016).

37. Cooper, H.M.: Organizing knowledge syntheses: A taxonomy of literature reviews. Knowl. Soc. 1, 104-126 (1988).

38. vom Brocke, J., Simons, A., Riemer, K., Niehaves, B., Plattfaut, R., Cleven, A.: Standing on the Shoulders of Giants: Challenges and Recommendations of Literature Search in Information Systems Research. Commun. Assoc. Inf. Syst. 37, (2015).

39. Roediger, H.L., Butler, A.C.: The critical role of retrieval practice in long-term retention. Trends Cogn. Sci. 15, 20-27 (2011).

40. Rowland, C.A.: The effect of testing versus restudy on retention: A meta-analytic review of the testing effect. Psychol. Bull. 140, 1432-1463 (2014).

41. Mayer, R.E.: Multimedia Learning. Cambridge University Press, Cambridge (2009).

42. Mayer, R.E., Moreno, R.: Nine Ways to Reduce Cognitive Load in Multimedia Learning. (2003).

43. Schwabe, G.;, Valerius, M.: Grundlagen des kollaborativen Lernens mit neuen Medien.

44. Andriessen, J., Baker, M.J., Suthers, D.: Arguing to Learn: Confronting Cognitions in Computer-Supported Collaborative Learning environments (2003).

45. Reznitskaya, A., Anderson, R.C., McNurlen, B., Nguyen-Jahiel, K., Archodidou, A., Kim, S.: Influence of Oral Discussion on Written Argument. Discourse Process. 155-175 (2001).

46. Scheiter, K., Gerjets, P.: Learner control in hypermedia environments. Educ. Psychol. Rev. 19, 285-307 (2007). 
47. Cohn, M.: User Stories Applied For Agile Software Development. (2004).

48. Jin, R.: The structure of pro and con arguments: A survey of the theories. (2011).

49. Fromm, H., Wambsganss, T., Söllner, M.: Towards a taxonomy of text mining features. 1$12(2019)$. 